\title{
FLOWERING IN PISUM. Hr, A GENE FOR HIGH RESPONSE TO PHOTOPERIOD
}

\author{
I. C. MURFET \\ Botany Department, University of Tasmania, Hobart, Australia
}

Received 12.ix.72

\section{Summary}

\begin{abstract}
LHR (late high response) peas are similar to $L$ (late) peas under long days but their flowering node is delayed to a much larger extent by short days. The LHR phenotype is conferred by dominant $H r$ in combination with $S n$. Addition of $E$ returns flower initiation to the early region (phenotype EI-early initiating). Since $L f$ is epistatic to $E$, genotype $L f E S n H r$ is also expected to be LHR. Hr has little effect by itself and $l f e s n \mathrm{Hr}$ is essentially ED (early developing) with some EI tendencies. $H r$ is in linkage group III 3 map units from the seed marbling gene $M$. Hr plants sometimes misclassify; lf $e \mathrm{Sn} \mathrm{Hr}$ as EI and lf e sn $\mathrm{Hr}$ as L. Cool temperatures reduce the effect of $\mathrm{Hr}$ but above $15^{\circ} \mathrm{lfe}$ $S n \mathrm{Hr}$ plants approach an obligate long day requirement for flowering. They can flower in response to a single inductive cycle. The flower inhibiting effect of $\mathrm{Sn}$, which short days favour, diminishes with age. It is suggested that $\mathrm{Hr}$ blocks the aging effect.
\end{abstract}

\section{INTRODUCTION}

Ar least six different phenotypic classes with respect to flowering behaviour may be recognised in Pisum by recording both node of first initiated flower and time of open flower under appropriate conditions of daylength (Murfet, $1971 a$ ). In a second paper in this series (Murfet, 1971b) three distinct phenotypic classes-ED (early developing), EI (early initiating) and L (late)were subjected to genetic analysis and it was shown that three dominant major genes $S_{1}, E$ and $S_{\mathrm{z}}$ determine between-class variation and also contribute substantially to within-class variation by combination and dosage effects. It was suggested that the historic symbols $L f$ and $S n$ be used in place of $S_{1}$ and $S_{2}$ respectively and $L f$ and $S n$ are used in the redefined sense here. A third paper (Murfet, 1971c) dealt with the possible physiological action of these genes. It was suggested that $S n$ produces a flower inhibitor in the cotyledons and shoot, that $E$ lowers the level of this inhibitor in the cotyledons and $L f$ increases the sensitivity of the apex to inhibitor.

A fourth phenotypic class, LHR (late high response), is now brought into the genetic analysis and it is shown that a fourth dominant major gene $H r$ will confer the ability to show a very large response to short day treatment given certain combinations of the other genes.

\section{Materials AND methods}

ED, EI and L plants may be distinguished under a photoperiod of 8 hours by recording the node at which the first flower bud is initiated and the time of first open flower. The classes are usually fully discrete under these conditions. ED plants flower early in node and time. EI plants initiate at an early node but because the development of the lower flower buds is either aborted 
or severely retarded under short days, flowering time is late. L plants flower late in node and time. LHR plants are indistinguishable from $\mathrm{L}$ plants under long days but their flowering is delayed to a much greater extent by short days. For example short days usually delay $\mathrm{L}$ plants by about $6-10$ nodes but a response of 25-50 nodes is usual in LHR plants. Further information on this system of phenotypic classification, which is based on the behaviour of standard varieties, and other information on growing conditions and scoring methods not covered below may be found in Murfet (1971a).

Parents, $F_{1}$ and other controls were grown in randomised blocks amongst the $F_{2}$. Data on flowering node and time were recorded only from main shoots. Node number is counted from the first scale leaf as one. Laterals, which have a tendency to grow from the lower nodes under short days, were regularly excised. Lateral branches also tend to grow from the higher nodes of LHR plants as they approach flowering. These were also excised in most cases but were sometimes allowed to grow when it was clear that flowers were already initiated on the main shoot. LHR plants may take over 7 months to complete their life-cycle and with 70-80 internodes even genetically dwarf (short internode length) plants may attain a height of over 2 metres. The cans containing these large plants were placed sufficiently far apart to allow light to reach the basal region and under these conditions even the lowest leaves remained green and healthy until well into the flowering phase.

Line $5 \mathrm{l}$ is essentially ED and a direct selection from the red-flowered, dwarf variety Vinco. Line 53 is an L type, shown previously to have genotype lf $e \mathrm{Sn}$. Lines 64 and 68 and the standard LHR variety Line 63, were selected from a cross between Lines 51 and 53 as indicated in the results section.

\section{RESULTS}

\section{(i) The gene $\mathrm{Hr}$}

Cross 21 between the early Line 51 and the late Line 53 gave rise to an $F_{1}$ whose flowering node was early and unaffected by photoperiod (table 1 ). A new type appeared in $F_{2}$ with the phenotype described above as LHR. To explain these results a fourth major gene $H r$, in addition to genes $L f, E$ and $S n$, is proposed to operate as follows. $H r$ by itself has little or no effect so that genotype lf $e$ sn $\mathrm{Hr}$ is early and essentially $\mathrm{ED}$ as is lf $E$ sn Hr. Hr in combination with $S n$ confers the ability to show a high response to short day treatment so that $l f e S n H r$ is LHR. Dominant $E$ remains epistatic to $S n$ in terms of flowering node even in the presence of $H r$ so that lf $E S n H r$ is EI.

Assuming genotype If $E$ sn $\mathrm{Hr}$ for Line 51, the cross with Line 53 (lf e Sn hr) should generate an EI $F_{1}$ and an $F_{2}$ ratio of 16 ED:36 EI:3 L:9 LHR. Unfortunately flowering time was not recorded for the $F_{1}$ and $F_{2}$, and ED and EI plants must be grouped under the general heading of early. (The present system of phenotypic classification based on flowering time as well as flowering node was adopted after the growing of Cross $2 \mathrm{I} \mathrm{F}_{2}$.) Cutting the $\mathrm{F}_{2}$ distribution in short days at node 17 and node 28 , the observed numbers of 117 early, $5 \mathrm{~L}$ and 22 LHR are in excellent agreement with expectation $\left(\chi_{2}^{2}=0 \cdot 77\right)$. Owing to pressure on space most $F_{2}$ plants were removed before reaching maturity but a few $F_{3}$ progenies were kept. The $F_{3}$ progeny given in table 1 row 7 showed a particularly clear-cut segregation into $7 \mathrm{ED}$, $2 \mathrm{~L}$ and $7 \mathrm{LHR}$ plants. These observed numbers were thought to represent a 


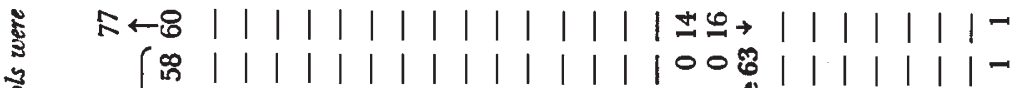

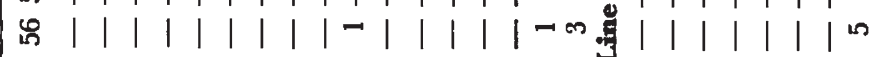

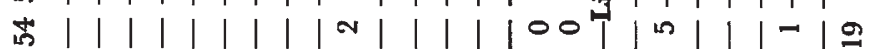

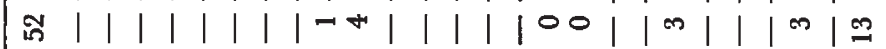

i $|1|||-\mid$ m $1|1| 100|101| 1010$



\& 1 | | | क्जा -

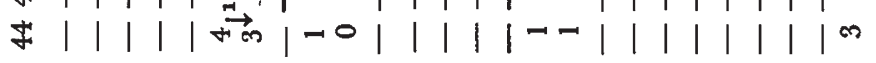

\% | | | | |m | mo | | | | I =N | | | | | | |

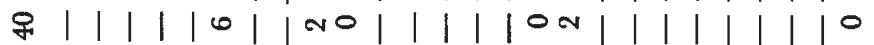

m | | | | | | | | n | | | |- | | | | | | |-

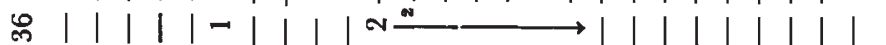

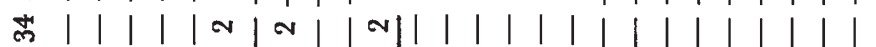

శ్ | | | | | N | | | m | | | | | | | | | | | | | |

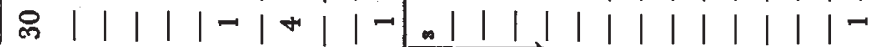

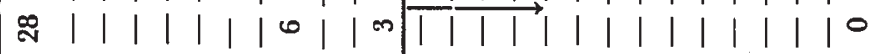

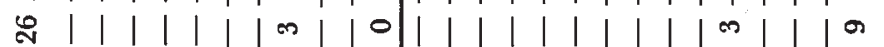

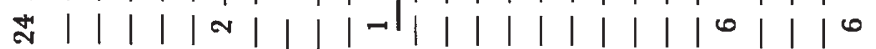

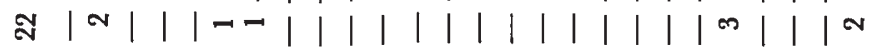



$\stackrel{\infty}{\infty}|1|-||||||||||||||||||-\infty$

$=1|1||||||||||||||||||||| n \mid$

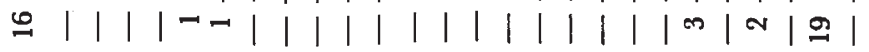

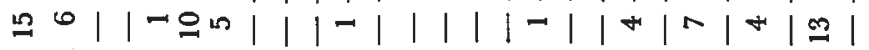



$\rightarrow-|m-\operatorname{mon}|-\longrightarrow||||||||||||-1$

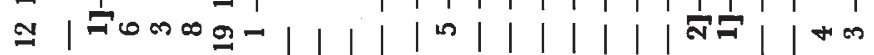

$=\mid$ |

을 |

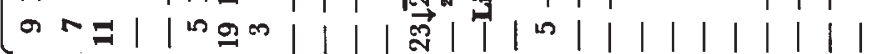

离

ตำ

范

का

边

능

bo 78

늘

उत्

总

要

نี

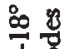

in

을

需

열

포

동 둥

Z

สิ

है

क्षे

కั

है.

窎.

$x$ ร

.

承 $\infty \propto \infty \infty \infty \infty \infty \infty \infty \infty \infty$

$\infty$

نं

논

สิ ถุ

容

总

告

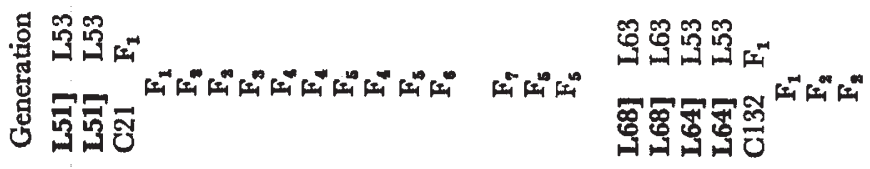




4 ED:3 L:9 LHR ratio and, therefore, a genotype of lflf ee Snsn Hrhr for the parental $\mathrm{F}_{2}$ plant. These conclusions were fully supported by an extensive analysis of the descendants of this $F_{8}$ progeny, as far as $F_{7}$ in some cases. For example the $7 \mathrm{ED}$ plants bred true in $\mathrm{F}_{4}$, one $\mathrm{L}$ plant gave a $1 \mathrm{ED}: 3 \mathrm{~L}$ ratio (Line 68, genotype lf e sn $h r$, is descended from an ED plant in this progeny) and the other L plant bred true apart from some EI plants which proved to be impenetrant $\mathrm{L}$ type (Impenetrance in genotype lf e $S n h r$ is dealt with by Murfet, 1971b). F from the LHR plants contained examples of all four possible segregation patterns, namely; 4 ED:3 L:9 LHR, 1 L:3 LHR, $1 \mathrm{ED}: 3 \mathrm{LHR}$ and all LHR. Examples of the latter two are given in table 1 where they and their descendents are used to illustrate "illegitimate " phenotypes in lf e Sn $\mathrm{Hr}$ and lf e sn $\mathrm{Hr}$ plants, temperature sensitivity in LHR plants, etc., dealt with below under the appropriate headings.

Row 11 contains an $\mathrm{F}_{4}$ progeny in exact agreement with the 1 ED:3 LHR ratio expected from an lflf ee Snsn HrHr plant. Line 64 is descended through these ED segregates and should therefore be homozygous for genotype If e sn Hr. To prove this genotype and substantiate the theory, Line 64 was crossed to Line 53 ( $l f e S n h r$ ) and the resulting data (Cross 132) are shown in the lower section of table 1. The strong interaction between $S n$ and $\mathrm{Hr}$, hidden in the $\mathrm{F}_{1}$ of Cross 21 by the epistasis of gene $E$, is fully revealed in Cross 132 where under short days the $F_{1}$ is some 28 nodes later than either parent and closely similar to Line 63 ( $l f e S n H r$ ). The observed $\mathrm{F}_{2}$ numbers in short days of $19 \mathrm{ED}, 21 \mathrm{~L}$ and $55 \mathrm{LHR}$ plants are in good agreement with an expected ratio of $4 \mathrm{ED}: 3 \mathrm{~L}: 9 \mathrm{LHR}\left(\chi_{2}^{2}=1 \cdot 56\right)$.

\section{(ii) Linkage of $\mathrm{Hr}$ and $\mathrm{M}$}

The Cross 132 data permit us to test the joint segregation of $\mathrm{Sn}$ and $\mathrm{Hr}$ with marker genes $A$ (anthocyanin pigmentation) and $M$ (marbling pattern on the testa) subject to the restrictions at the foot of table 2. Segregation for $S n$, often obscured in long days, can be followed in Cross $132 \mathrm{~F}_{2}$ because of the very marked bimodality of the continuous distribution. sn plants were taken as flowering at node 13 or earlier. Individual segregation data for $A, M, S n$ and $H r$ are all normal. $S n$ was shown previously to segregate freely

TABLE 2

Joint segregation data for $\mathrm{A}, \mathrm{M}, \mathrm{Sn}$ and $\mathrm{Hr}$ obtained from the $F_{2}$ of

Cross 132 (A M sn $\mathrm{Hr} \times$ a m Sn hr)

\begin{tabular}{|c|c|c|c|c|c|c|c|c|}
\hline & $\begin{array}{r}\text { Phe } \\
\text { observ }\end{array}$ & $\begin{array}{l}\text { type } \\
\text { numk }\end{array}$ & & Total & Gene & Gene & Joint & Recombination \\
\hline $\begin{array}{c}A S n \\
93\end{array}$ & $\begin{array}{c}A s n \\
24\end{array}$ & $\begin{array}{c}a S n \\
21\end{array}$ & a sn & 143 & $A_{3.55}^{A^{11}}$ & $\begin{array}{c}S n \\
1.70\end{array}$ & 0.01 & \\
\hline $\begin{array}{c}M S n \\
70\end{array}$ & $\begin{array}{c}M s n \\
17\end{array}$ & $\begin{array}{c}m S n \\
22\end{array}$ & $\begin{array}{c}m s n \\
6\end{array}$ & 115 & $\begin{array}{c}M \\
0.03\end{array}$ & $\begin{array}{c}S n \\
1.53\end{array}$ & 0.05 & \\
\hline $\begin{array}{c}A H r \\
46\end{array}$ & $\begin{array}{c}A h r \\
15\end{array}$ & $\begin{array}{c}a r \\
9\end{array}$ & $\begin{array}{r}a h r \\
5\end{array}$ & 75 & $\underset{1 \cdot 60}{A}$ & $\begin{array}{l}H r \\
0 \cdot 11\end{array}$ & 0.53 & \\
\hline$\underset{44}{M H r}$ & $\begin{array}{c}M h r \\
0\end{array}$ & $\begin{array}{c}m H r \\
2\end{array}$ & $\begin{array}{c}m h r \\
15\end{array}$ & 61 & $\begin{array}{c}M \\
0.27\end{array}$ & $\begin{array}{c}H r \\
0.01\end{array}$ & $54 \cdot 52$ *** & $3.3 \% \pm 2.3 \%$ \\
\hline
\end{tabular}

The segregation of $\mathrm{Hr} / \mathrm{hr}$ could only be followed in $S n$ plants under short days and the segregation of $M / m$ could only be followed in $A$ plants. $* * \mathrm{P}<0.001$. 
with $A, I, C y_{1}, V, P$ and $R$ (Murfet, 1971b), and $M$ may now be added to the list. $H r$ showed free recombination with $A$ but tight linkage with $M$ (RCV 3 per cent.) and is therefore in linkage group III (groups after Lamprecht, 1961). The free recombination between $A$, and $S n$ and $\mathrm{Hr}$ provides added evidence that we are not dealing here with a variant of the third latening gene, $L f$, which shows a firm linkage with $A$.

\section{(iii) "Illegitimate" phenotypes for lf e $\mathrm{Sn} \mathrm{Hr}$ and if e sn $\mathrm{Hr}$}

Rows 8 and 9 of table 1 are samples from the same $F_{4}$ progeny which is undoubtedly homozygous if e $S_{n} H r$. However, one plant was phenotypically EI flowering at node 13 and showing under the short day conditions suppressed floral development and prolonged growth. The great majority of its $F_{5}$ progeny are LHR (row 10 , table I) thereby revealing its true genetic nature. It may therefore be considered as "illegitimate" EI or impenetrant LHR. The same phenomenon was observed in other instances with this genotype as well as cases in which the illegitimate EI plant proved to be genetically lflf ee SnSn Hrhr or lflf ee Snsn Hrhr.

The other proven case of misclassification concerns genotype lf e sn $\mathrm{Hr}$ which normally classifies ED but which occasionally flowers around node 18 or 20 thus classifying with the L class. An example may be seen in the $\mathrm{F}_{8}$ progeny in row 13 of table 1 . The ancestry and descendants of the plant flowering at node 20 establish the genotype as pure If e sn $\mathrm{Hr}$. The illegitimate nature of this plant was also indicated by a more limited growth pattern and lower yield than is usual for legitimate $L$ plants. One $F_{2}$ plant of Cross 132 flowering at node 18 in short days appeared to be of this type and $F_{3}$ data confirmed its illegitimacy. It has been grouped with the $s n$ plants in table 2 .

\section{(iv) Temperature and flowering in if e $\mathrm{Sn} \mathrm{Hr}$}

The influence of temperature was not deliberately studied in the present work but it became apparent that even under 8-hour short days cooler night temperatures (e.g. $12^{\circ} \mathrm{C}$.) may cause the LHR plants to flower earlier and intergrade with the L class. This effect is illustrated in table 1 by samples of the same pure LHR progeny grown at night temperature $11^{\circ}-14^{\circ} \mathrm{C}$. (row 8) and night temperature $15^{\circ}-18^{\circ} \mathrm{C}$. (row 9 ). The lowest of these temperatures is still well above those used in vernalisation studies. We have maintained LHR plants under 8-hour short days with $18^{\circ} \mathrm{C}$. nights for as long as 4 months, but the plants have remained vegetative until transferred to long days (over 70 nodes have been laid down by this time), suggesting that at warmer temperatures LHR plants may have an obligate long-day requirement for flowering.

\section{(v) Response of lf e $\mathrm{Sn} \mathrm{Hr}$ to a single inductive cycle}

The LHR plants in rows 16 and 17 of table 1 were inadvertently exposed to a single long-day cycle when they had expanded approximately 30 leaves. Such plants have a further 10-12 nodes already laid down in the apex, and it would seem that some 40 per cent. of the plants were induced to flower by this single inductive cycle as they have commenced flowering around node 42. The rest of the plants went on to flower at a much higher node giving rise to the bimodal distribution of the LHR class. A second sample of these same progenies not exposed to the inductive cycle gave a compact, unimodal LHR class. Reid (unpublished), of this department, has subsequently 
shown that Line 63 can be induced to flower when a short day cycle of 8 hours light/ 16 hours dark is broken by the sequence 12.5 hours light/ 6 hours dark/ 13.5 hours light.

(vi) Phenotypic comparison of the genotypes if e sn hr and lf e sn $\mathrm{Hr}$

Genotype If esn $\mathrm{Hr}$ has been treated so far as having a normal ED phenotype. However, by comparing appropriate segregates and pure lines an attempt was made to recognise any phenotypic effects gene $\mathrm{Hr}$ may have when present on an lfe sn background. With good growing conditions under either short or long days $H r$ and $h r$ plants were reasonably close in flowering node and flowering time but $H r$ plants had a tendency to give a higher yield and a very definite tendency to continued growth under short days. The latter features trend towards the EI phenotype and this trend is further evident under less favourable short-day conditions (higher night temperature and/or weaker light intensity) where the $H r$ plants abort their lower flower buds more readily than $h r$ plants. These features are partially illustrated in table 3 using plants grown under the weak light intensity of

Table 3

Node of first initiated flower (FI), days to open flower (FT), total number of nodes expanded (TN) and number of seeds ( $Y_{\text {ield }}$ ) for genotype if e sn $\mathrm{Hr}$ and ED, EI and $L$ plants. Photoperiod 8 hours for the first 3 months; thereafter long days. Night temperature $15^{\circ}-18^{\circ} \mathrm{C}$

\begin{tabular}{|c|c|c|c|c|c|c|c|c|c|c|c|}
\hline \multirow[b]{2}{*}{ Line } & \multirow[b]{2}{*}{ Genotype } & \multirow[b]{2}{*}{ Phenotype } & \multicolumn{2}{|c|}{ FI } & \multicolumn{2}{|c|}{ FT } & \multicolumn{2}{|c|}{$\mathrm{TN}$} & \multicolumn{2}{|c|}{ Yield } & \\
\hline & & & $\bar{x}$ & S.E. & $\bar{x}$ & S.E. & $\bar{x}$ & S.E. & $\bar{x}$ & S.E. & \\
\hline & lfesn hr & $\mathrm{ED}$ & $10 \cdot 25$ & 0.13 & $39 \cdot 8$ & 0.6 & $16 \cdot 0$ & 0.4 & $4 \cdot 2$ & 0.4 & 12 \\
\hline & lf esn $\mathrm{Hr}$ & - & $10 \cdot 83$ & 0.17 & 52.9 & 1.4 & $24 \cdot 3$ & 0.8 & $10 \cdot 0$ & 0.9 & 12 \\
\hline & If $E S n h r$ & EI & $12 \cdot 25$ & 0.22 & $71 \cdot 6$ & 0.7 & 34.5 & $1 \cdot 3$ & $53 \cdot 4$ & $5 \cdot 0$ & 12 \\
\hline & lf e Sn hr & L & $24 \cdot 64$ & 0.34 & 77.0 & $1 \cdot 3$ & $36 \cdot 8$ & 0.8 & $39 \cdot 1$ & $2 \cdot 8$ & 11 \\
\hline & lf esn hr* & $\mathrm{ED}$ & $10 \cdot 60$ & 0.24 & $44 \cdot 0$ & 0.8 & $13 \cdot 4$ & 0.7 & $4 \cdot 6$ & $2 \cdot 4$ & \\
\hline $32 \mathrm{~F}$ & lfe sn $H_{r}-*$ & - & $11 \cdot 30$ & 0.15 & $54 \cdot 2$ & $2 \cdot 5$ & $20 \cdot 1$ & $1 \cdot 2$ & $7 \cdot 1$ & 1.4 & 10 \\
\hline
\end{tabular}

* These plants are respectively the early $A m$ and $A M$ segregates, the tight linkage between $M$ and $H r$ being used to assign a probable genotype for $H r$.

mid-winter. Although the lf e sn $\mathrm{Hr}$ plants are intermediate between the standard ED habit (represented here by lf e sn $h r$ plants) and the standard EI plants (line 60) in respect of all four variables, they are closer in every case to the ED type. Therefore, although it may be possible to find environmental conditions which will allow if e sn $\mathrm{Hr}$ plants to be distinguished fairly accurately from standard ED plants when several developmental characters are considered, the general similarities between the forms appear strong enough to justify the continued placement of $l f e s n \mathrm{Hr}$ in the ED class.

\section{Discussion}

Flowering has been considered to be under polygenic or largely polygenic control by some workers (e.g. Rowlands, 1964; Watts et al., 1970). Certainly under typical temperate field conditions of long days and cool nights flowering variation is often quantitative with little hint of underlying Mendelian genes. However, by choosing appropriate environmental conditions to expose segregation, taking account of more than one aspect of gene expression and following crosses for a number of generations, it has proved possible to 
recognise and isolate four major genes controlling flowering (three have been located). The present investigations also indicate the occurrence of polygenic systems (Murfet, 1971b), but just as the four genes $L f, E, S n$ and $H r$ have quantitative effects in some circumstances it is possible that more refined investigatory techniques may expose further Mendelian genes amongst systems presently treated as quantitative.

Although classic Mendelian patterns may be obtained even the use of special environments will not necessarily give absolute resolution in all cases. For example, short days have broken the $\mathrm{F}_{2}$ of Cross 21 into several discrete classes but the early portion of the $F_{2}$ (say nodes 9-17) may still contain all 27 possible genotypes including six illegitimate occupants (lflf ee $\mathrm{Sn}$ - $h \mathrm{rhr}$ and lflfee $S_{n}-H r$-) and the ED/EI difference may be blurred in this complex cross by the deviation of some ED forms (e.g. lf e sn Hr) toward the EI phenotype. In the latter case yield may be used as an aid to classification but whilst substantial differences in mean yield occur between $S n$ and $s n$ plants, individual plants may have completely anomalous values as a result of sporadic fungal or root fly attack, etc.

The possibility of a fifth locus is raised by the work of Marx $(1968,1969)$. If his $I$ and $G_{2}$ phenotypic classes are identical to ED and EI respectively then the present four loci provide no equivalent to his case of complementary genes for sensitivity to photoperiod. On the other hand there is some evidence (not yet conclusive) that lf $E S n \mathrm{Hr}$ plants have a greater tendency to continued growth under short days than lf $E S n h r$ plants. Both classify as EI but if the former corresponds to $G_{2}$ and the latter classifies as $I$ under the growing conditions and slightly longer photoperiods used by Marx, then the cross lf $E$ Sn $h r(\mathrm{I}) \times$ lf $E$ sn $H r(\mathrm{I})$ would give a $\mathrm{G}_{2} \mathrm{~F}_{1}$ and a $9 \mathrm{G}_{2}$ : 7 I ratio in $\mathrm{F}_{2}$. Bremer and Weiseth (1961) crossed a pea showing a very large response to photoperiod (probably an LHR variety) with a day neutral type and found a single gene difference, the day neutral form being recessive for a gene which they named $d n$. Most of the information which they give is consistent with the view that their cross was segregating for the $S n / s n$ pair of alleles on an lf $\mathrm{H} \mathrm{Hr}$ background. However, the question of relationship between various varieties can best be resolved by intercrossing and direct comparison.

Concerning the possible physiological action of gene $\mathrm{Hr}$, it may be noted that $\mathrm{Hr}$ has very little effect by itself under long days, but causes a very marked prolongation of the vegetative state under short days when present in combination with $\mathrm{Sn}$. $\mathrm{Hr}$ is therefore modifying the expression of $S n$. There is evidence (Murfet, $1971 b, c$ ) that $S n$ is responsible for the production of a substance which delays flower initiation, retards floral development and opposes senescence and that $S n$ activity inevitably diminishes with age. One possible action of $\mathrm{Hr}$ may be to increase the level of output from the $\mathrm{Sn}$ gene. This suggestion is opposed by the fact that lf e $\mathrm{Sn} \mathrm{Hr}$ and lf e Sn hr plants both show occasional premature flowering (illegitimate EI) indicating that the $\mathrm{Hr}$ plants are not necessarily deeper in the vegetative state. It seems more likely that $H r$ blocks the effect of ageing on $S n$ so that $S n$ activity continues indefinitely under short days. The absence of an $\mathrm{Hr}$ effect in lf $e \mathrm{Sn}$ plants under long days is consistent with this theory if $S n$ is assumed to be inactive under long days. This assumption may appear negated by the fact that under long days $l f e S n$ plants are a few nodes later than lf e sn plants but the cotyledons are normally underground in the dark and in addition Paton (1971) has shown that the cotyledons are insensitive to photoperiod for the first 4 
days. Absence of $S n$ activity in long days could be tested by growing excised embryos of lf $e s n h r$ and lf $e S n h r$ plants which would be expected to flower at the same node in continuous light given identical polygenic backgrounds. It seems therefore that age, photoperiod and gene $\mathrm{Hr}$ may be regulating $S n$ activity at the gene level and we are currently investigating the possible involvement of phytochrome in this regulatory system.

The promotory effect of cool temperatures on flowering in LHR plants is probably mediated through $S n$ not $H r$ as such effects are well known in L-type peas (Haupt, 1969). The response is more strikingly manifest in LHR plants because they have a much wider potential range of flowering behaviour than $\mathrm{L}$ plants. The light effect of $\mathrm{Hr}$ on continued growth, etc., of $l f e s n$ plants under short days could also be mediated via the $s n$ locus if, as previously suggested (Murfet, 1971c), recessive $s n$ is a leaky mutant.

Genotype $L f E S n H r$ is not described here but since $L f$ has so far proved epistatic to $E$, an LHR phenotype would be expected. Like Cross 132, a cross between $L f e S n h r$ and lf $E$ sn $H r$ could provide an example of an $\mathrm{F}_{1}$ much later than either parent. The qualitative response to photoperiod obtainable with lf e Sn $\mathrm{Hr}$ plants and their sensitivity to temperature and to a single inductive cycle should make these plants a useful tool for the further study of the physiological-genetics of flowering.

\footnotetext{
Acknowledgments.- This work was supported in part by a grant from the Australian Research Grants Committee.
}

\section{REFERENGES}

BREMER, A. H., AND WEISETH, G. 1961. Problems of vegetable production in relation to daylength on latitudes 58-70 N. In: J. Garnaud (ed.), Adv. hort. sci. and their applications, pp. 426-435. Proc. XVth int, hort. cong. Nice 1958, v. 1. Pergamon Press, New York. HAUPT, w. 1969. Pisum sativum. In: L. T. Evans (ed.), The Induction of Flowering: Some Case Histories, pp. 393-408. Macmillan and Co., Melbourne.

I.AMPrecht, H. 1961. Die Genenkarte von Pisum. Agri Hort. Genet., 19, 360-401.

MARX, G. A. 1968. Influence of genotype and environment on senescence in peas Pisum sativum L. BioScience, 18, 505-506.

MarX, G. A. 1969. Some photo-dependent responses in Pistm. I. Physiological behaviour. Crop. Sci., 9, 273-276.

MURFet, 1. c. 1971a. Flowering in Pisum. Three distinct phenotypic classes determined by the interaction of a dominant early and a dominant late gene. Heredity, 26, 243-257.

MURFET, I. c. 1971b. Flowering in Pisum. A three-gene system. Heredity, 27, 93-110.

MURFET, I. c. 1971c. Flowering in Pisum. Reciprocal grafts between known genotypes. Aust. F. biol. Sci., 24, 1089-1101.

PATON, D. M. 1971. Photoperiodic induction of flowering in the late pea cultivar Greenfeast: the role of exposed cotyledons and leaves. Aust. F. biol. Sci., 24, 609-618.

Rowlands, D. G. 1964. Genetic control of flowering in Pisum sativum L. Genetica, 35, 75-94. WATTS, L. E., STEVENSON, E., AND GRAMPTON, M. J. 1970. Inheritance of flowering time in six pea cultivars (Pisum sativum L.). Euphytica, 19, 405-410. 